Bone. 2014 March ; 60: 235-245. doi:10.1016/j.bone.2013.12.023.

\title{
The Rho-GEF Kalirin regulates bone mass and the function of osteoblasts and osteoclasts
}

\author{
Su Huang ${ }^{\mathrm{a}}$, Pierre P. Eleniste ${ }^{\mathrm{a}}$, Kornchanok Wayakanon ${ }^{\mathrm{a}}$, Prashant Mandela ${ }^{\mathrm{b}}$, Betty A. \\ Eipper $^{\mathrm{b}}$, Richard E. Mains ${ }^{\mathrm{b}}$, Matthew R. Allen ${ }^{\mathrm{c}}$, and Angela Bruzzaniti ${ }^{\mathrm{a}, \mathrm{c},{ }^{*}}$ \\ aDepartment of Oral Biology, Indiana University School of Dentistry, Indianapolis, IN, USA \\ ${ }^{b}$ Department of Neuroscience, University of Connecticut Health Center, Farmington, CT, USA \\ 'Department of Anatomy and Cell Biology, Indiana University School of Medicine, Indianapolis, \\ IN, USA
}

\begin{abstract}
Bone homeostasis is maintained by the balance between bone resorption by osteoclasts and bone formation by osteoblasts. Dysregulation in the activity of the bone cells can lead to osteoporosis, a disease characterized by low bone mass and an increase in bone fragility and risk of fracture. Kalirin is a novel GTP-exchange factor protein that has been shown to play a role in cytoskeletal remodeling and dendritic spine formation in neurons. We examined Kalirin expression in skeletal tissue and found that it was expressed in osteoclasts and osteoblasts. Furthermore, micro-CT analyses of the distal femur of global Kalirin knockout (Kal-KO) mice revealed significantly reduced trabecular and cortical bone parameters in Kal-KO mice, compared to WT mice, with significantly reduced bone mass in 8,14 and 36 week-old female Kal-KO mice. Male mice also exhibited a decrease in bone parameters but not to the level seen in female mice.

Histomorphometric analyses also revealed decreased bone formation rate in 14 week-old female Kal-KO mice, as well as decreased osteoblast number/bone surface and increased osteoclast surface/bone surface. Consistent with our in vivo findings, the bone resorbing activity and differentiation of Kal-KO osteoclasts was increased in vitro. Although alkaline phosphatase activity by Kal-KO osteoblasts was increased in vitro, Kal-KO osteoblasts showed decreased mineralizing activity, as well as decreased secretion of OPG, which was inversely correlated with ERK activity. Taken together, our findings suggest that deletion of Kalirin directly affects osteoclast and osteoblast activity, leading to decreased OPG secretion by osteoblasts which is likely to alter the RANKL/OPG ratio and promote osteoclastogenesis. Therefore, Kalirin may play a role in paracrine and/or endocrine signaling events that control skeletal bone remodeling and the maintenance of bone mass.
\end{abstract}

\footnotetext{
(C) 2013 Elsevier Inc. All rights reserved.

*Corresponding author at: Indiana University School of Dentistry, Department of Oral Biology DS241, 1121 W. Michigan Street, Indianapolis, IN 46202, USA. Fax: +1 317278 1411. abruzzan@iu.edu (A. Bruzzaniti).

Supplementary data to this article can be found online at http://dx.doi.org/10.1016/j.bone.2013.12.023.

Authors' roles: Study conception and design; SH, PE, REM, MRA and AB. Acquisition of data; SH, PE, MRA, KW and AB. Analysis and interpretation of data; SH, PE, BAE, REM, MRA and AB. Drafting of manuscript and revising it critically for important intellectual content: SH, PE, BAE, REM, MRA and AB. Approved final version of manuscript: all authors. AB takes responsibility for the integrity of the data analysis.
}

Disclosures

The authors state that they have no conflicts of interest. 


\section{Keywords}

GTP-exchange factor; Receptor activator of nuclear factor $\mathrm{kb}$ ligand; Osteoprotegerin, bone remodeling

\section{Introduction}

Bone remodeling occurs through the coordinated activities of osteoclasts, osteoblasts and osteocytes, which orchestrate osteoclast and osteoblast activities in response to changes in mechanical stimuli or hormonal levels. Osteoclastogenesis is tightly regulated by local and circulating levels of macrophage-colony stimulating factor (M-CSF), receptor activator of NF- $\kappa \mathrm{B}$ ligand (RANKL) and osteoprotegerin (OPG), a decoy receptor of RANKL. Dysregulated bone cell activity can lead to bone loss and osteoporosis, a major health issue characterized by low bone mass, micro-architectural deterioration of bone with a consequent increase in bone fragility and risk of fracture. Although several treatment options exist to prevent or decrease bone loss, such as bisphosphonates, denosumab, raloxifene and teraperatide, adverse side effects have been associated with each of these treatment modalities [1], necessitating a continued search for alternatives.

The molecular regulators of bone cell function and the crosstalk between bone cells during bone remodeling are poorly understood. RhoGTPases such as Rho, Rac and Cdc 42 act as molecular switches in a number of intracellular signal pathways and are involved in the regulation of cell differentiation and proliferation, cytoskeleton remodeling, vesicle trafficking and nuclear transport [2,3]. Rac1 has been previously shown to play a role in osteoclastogenesis and osteoclast motility [3]. Moreover, Rac2 knockout mice have increased trabecular bone mass and osteoclasts lacking Rac2 exhibit defective chemotaxis and bone resorbing activity [3]. The Rho-GTPases are activated by the guanine nucleotide exchange factors (GEFs), which convert inactive Rho-GDP into active Rho-GTP [3,4], yet it is unclear how the expression and function of the Rho-GEFs affect bone cell function.

Kalirin is a recently identified dual Rho-GEF belonging to the family of Dbl-homology (DH) proteins; an N-terminal GEF activates Rac1 while a C-terminal GEF activates RhoA $[5,6]$. Kalirin mRNA isoforms are generated by different transcription initiation sites and alternative splicing. The most abundant isoforms are Kalirin-7, Kalirin-9 and Kalirin-12. The largest isoform, Kalirin12, contains an $\mathrm{N}$-terminal Sec14 lipid binding domain, nine tandem spectrin repeats, two Rho-GEF (PH-DH) domains, two SH3 domains, an Ig/FnIII domain and a Ser/Thr kinase domain (Fig. 1A). Kalirin has been shown to play a role in cytoskeletal remodeling, synapse and dendritic spine morphogenesis and extension, secretory granule maturation and plasticity of dendritic spines in neuronal cells [7-11]. Recently, Kalirin has also been shown to be important in neuromuscular function [7-12]. Given that the activity of Rho-GTPases is critical for the function of osteoclasts and osteoblasts, we hypothesized that Kalirin may be important for the regulation of bone cells and bone mass. In the current study, we used Kalirin global knockout (Kal-KO) mice to investigate the role of Kalirin in osteoblast and osteoclast function, and its effect on trabecular and cortical bone mass acquisition and maintenance.

\section{Materials and methods}

\section{Preparation of neonatal calvarial osteoblastic cells and bone marrow stromal osteoblasts}

Murine calvarial osteoblasts were prepared as previously described [13]. 1-3 day old mouse pups were sacrificed and tails kept for genotyping. Calvaria were freed of tissue and incubated in digestion buffer containing $0.1 \%$ collagenase $\mathrm{A}$ and $0.2 \%$ dispase (Roche) 
dissolved in serum free a-MEM for $10 \mathrm{~min}$ at $37^{\circ} \mathrm{C}$. Undigested calvaria were allowed to settle and digestions were repeated 3-5 times, each for $15 \mathrm{~min}$. The supernatant from the first digestion was discarded, but all others were collected, pooled and centrifuged. Calvarial osteoblasts were grown in a-MEM $+10 \%$ FBS for up to 7 days, with one passage at day 3 , before being used for experiments. For some in vitro experiments, charcoal-dextran stripped fetal bovine serum (CH-FBS) was used, which contains low levels of estradiol, cortisol, corticosterone, the B vitamins, T3, T4 and prostaglandins as described by the manufacturer (Gemini Bio-products, \#100-119). To isolate bone marrow-derived osteoblasts, bone marrow was flushed from mice and cultured for 2 days. Non-adherent cells were removed by extensive washing. Attached cells were grown for 1 week until colonies formed. The cells were then tyrpsinized and cultured in osteogenic media for up to 28 days.

\section{Preparation of osteoclasts}

Authentic osteoclasts (OC) were isolated from the bone marrow of 6-8 week old C57/B16 mice (Jackson Labs, ME). Osteoclast-like cells were differentiated from bone marrow in the presence of calvarial-derived osteoblasts plus $10^{-8} \mathrm{M} \mathrm{VitD}_{3}$ and $10^{-6}$ PGE2 (co-culture system) or by treatment with $20 \mathrm{ng} / \mathrm{ml}$ M-CSF and $80 \mathrm{ng} / \mathrm{ml}$ RANKL (Peprotech) as previously described $[5,6,14,15]$. Osteoclast bone resorbing activity was measured by replating mature osteoclasts on dentin (Nordic biosciences) for $48 \mathrm{~h}$. Osteoclasts were removed and the degraded area was stained with toluidine blue and the area quantified using the Bioquant analysis software (Nashville, TN). The area resorbed was normalized for the number of multinucleated cells expressing tartrate-resistant acid phosphatase (TRAP).

\section{Quantitative measurement of alkaline phosphatase activity}

Osteoblasts were plated at $2 \times 10^{6}$ cells per $1.9 \mathrm{~cm}^{2}$ well and grown for 7 days in osteogenic media containing $10 \mu \mathrm{M}$ ascorbic acid and $50 \mu \mathrm{M} \beta$-glycerophosphate. Cells were washed with PBS, and lysed in $1 \%$ TritonX-100 supplemented with protease/kinase inhibitors, subjected to freeze-thaw cycles, and lysates were collected by centrifugation. Alkaline phosphatase activity (ALP) was then determined by the colorimetric conversion of $\mathrm{p}$ nitrophenol phosphate to nitrophenol (Sigma) following published procedures. Lysates were incubated with $100 \mu \mathrm{l}$ of $3 \mathrm{mg} / \mathrm{ml}$ substrate solution for $30 \mathrm{~min}$ at $37^{\circ} \mathrm{C}$ and the reaction stopped with $20 \mathrm{mM} \mathrm{NaOH}$. Optical absorbance at $405 \mathrm{~nm}$ was read in a Thermax multiplate reader. ALP activity was normalized by total protein per well as determined using the BCA protein assay kit (Pierce).

\section{Quantitative analysis of mineralization}

Calcium deposition was measured by eluting Alizarin Red S stain from osteoblasts differentiated with ascorbic acid and $\beta$-glycerophosphate for 7-14 days. Prior to staining, cells were washed four times with PBS, and then fixed with ice-cold $70 \%$ ethanol at $-20{ }^{\circ} \mathrm{C}$ for $1 \mathrm{~h}$. Cells were allowed to warm up to room temperature for $10 \mathrm{~min}$, and then washed two times with distilled water. Cells were stained with $40 \mathrm{mM}$ Alizarin Red-S solution (pH 4.2) at room temperature with shaking for $10 \mathrm{~min}$, and subsequently washed 5 times with distilled water, and once with PBS. Bound Alizarin Red was eluted by adding $1 \%$ acetylpyridinium chloride (Sigma \#C0732) in $10 \mathrm{mM}$ sodium phosphate (pH 7.0) to wells for $15 \mathrm{~min}$ at room temperature. The absorption of the eluted solution was read at $562 \mathrm{~nm}$. The amount of calcium in solution was calculated based on the known ability of Alizarin Red to bind 2 mol of calcium per mol of dye in solution.

\section{RNA isolation and reverse-transcription PCR}

Total RNA was isolated from samples using the Qiagen RNA mini prep Kit (\#74105). Total RNA was treated with DNase I (Ambion \#Am2222l) at room temperature for $15 \mathrm{~min}$ 
followed by the addition $25 \mathrm{mM}$ EDTA ( $10 \%$ total volume) and heat inactivation at $65{ }^{\circ} \mathrm{C}$ for $10 \mathrm{~min} .500 \mathrm{ng}$ of DNase I treated total RNA was used in reverse transcriptions using the First Strand cDNA kit (Roche \#04379012001). Random hexamers were used as primers. The resulting cDNA were then used as the template for PCR amplification (Thermo Scientific AB-0192/A). The PCR reaction was initiated by denaturing the DNA at $94{ }^{\circ} \mathrm{C}$ for 4 min followed by 28 cycles of denaturing at $94{ }^{\circ} \mathrm{C}$ for $30 \mathrm{~s}$, annealing at $58{ }^{\circ} \mathrm{C}$ for $30 \mathrm{~s}$ and extension at $72{ }^{\circ} \mathrm{C}$ for $1 \mathrm{~min}$. The final cDNA extension step was performed at $72{ }^{\circ} \mathrm{C}$ for 7 $\mathrm{min}$. The amplified fragments were separated on $2.0 \%$ agarose gels, visualized by ethidium bromide staining and imaged using a Bio-Rad Gel Doc XR imaging system.

\section{Western immunoblotting}

Cells were washed twice with cold PBS and lysed in buffer containing $62.5 \mathrm{mM}$ Tris-HCl, $\mathrm{pH} 6.8,2 \% \mathrm{w} / \mathrm{v}$ SDS, $10 \%$ glycerol supplemented with protease inhibitors (100 $\mu \mathrm{M}$ PMSF, $0.15 \mu \mathrm{M}$ aprotinin, $1 \mu \mathrm{M}$ leupeptin and $1 \mu \mathrm{M}$ pepstatin). Lysates were sonicated for $10 \mathrm{~s}$ and clarified by centrifugation. Proteins were resolved by SDS-PAGE electrophoresis and transferred to nitrocellulose membrane. Western blotting with antibodies to ERK, actin or Kalirin (07-122, Millipore) was performed using standard methodology and the proteinantibody complexes were visualized using the enhanced chemiluminescence system (Pierce). Long bones from WT and Kal-KO mice were washed free of tissue and bone marrow flushed with PBS. Bones were crushed and proteins were extracted in T-Per solution (Pierce) containing phosphatase inhibitors. Quantitation of protein band intensity was carried out by densitometry using the NIH Image program htpp://rsb.info.nih.gov/nihimage.

\section{Generation of Kalirin-deficient mice}

Kalirin spectrin repeat knockout (Kal-KO) mice were generated and validated [12], and a colony was established at Indiana University School of Dentistry. All mouse work was carried out under IACUC protocol DS000885R. Briefly, exon 13 of the Kalrn gene encodes the $\mathrm{N}$-terminal spectrin repeat region common to all major Kalirin isoforms [5,6]. Kalrn exon 13 floxed mice (flanked with loxP sites) were crossed with mice expressing the Crehypoxanthine guanine phosphoribosyl transferase (Hprt) mice, to drive global germ-line expression of Cre (Jackson Labs no. 004302). Exon 13 of the Kalrn gene encodes an exon in the spectrin repeat region common to all major Kalirin isoforms $[5,6]$. The major isoforms Kalirin-7, -9 and -12 are ablated in these mice, although minor transcripts containing only the second GEF domain can be produced from a remaining downstream promoter. All mice were congenic on the C57/B16 background and were backcrossed greater than 15 generations. Male and female Kal-KO and WT littermate mice at 8, 14 and 36 weeks of age were used for skeletal analyses and serology. Genotyping was performed by tail snip and RT-PCR using primers spanning the loxP sites (Fig. 1C) (primer A, TGTATGCCTTGGAAACAGGC; primer B, TGTTTTGCCATCGGGAGGAT; primer C, CGCAAGGAACATCGGGCTTT). GAPDH was used as an internal control (forward primer; 5'-AACTTTGGCATTGTGGAAGG-3'; reverse primer: 5'CCCTGTTGCTGTAGCCGTAT-3').

\section{Serum collection and analysis}

Whole blood from 14 week old mice was collected by cardiac puncture at necropsy and kept at room temperature for $30 \mathrm{~min}$ and transferred to $4{ }^{\circ} \mathrm{C}$ overnight. The coagulated blood was centrifuged at $4000 \mathrm{rpm}$ for $20 \mathrm{~min}$. Serum was carefully transferred into a new sterile tube and aliquots stored at $-80^{\circ} \mathrm{C}$. Hormone and cytokine levels in serum were quantified using commercial ELISA kits as follows: osteoprotegerin (OPG, RayBio \#ELM-OPG-001); receptor activator of NF- $\kappa$ B (RANKL RayBio \#ELM-TRANCE-001); TRAP (TRACP5b, 
IDS \#SB-TR103), fragment of type I collagen (CTX, IDS \#AC-06F1), amino pro-peptide of type 1 collagen (P1NP, IDS \#AC-33F1), and osteocalcin a serum biomarker of bone formation (OCN, Invitrogen \#KAQ1381). Insulin-like growth factor-1 (IGF-1) was measured using an ELISA kit from IDS.

\section{Micro-computed tomography}

Micro-computed tomography (micro-CT) was performed using a Skyscan 1172 highresolution desk-top system as previously reported [16] following standard nomenclature. Briefly, distal femora were scanned using an X-ray source set at $60 \mathrm{kV}$ with a $6 \mu \mathrm{m}$ pixel size. The trabecular bone compartment was segmented from the cortical shell for 50 slices in a region $\sim 0.5 \mathrm{~mm}$ proximal to the most distal portion of the growth plate for each animal. Cortical bone analyses on a single slice were conducted at a site $2 \mathrm{~mm}$ proximal from the most distal region of the trabecular bone region of interest.

\section{Histomorphometry}

Tissue for histology was embedded in methyl methacrylate and mid-sagittal $(4 \mu \mathrm{m})$ sections of cancellous bone from the distal femur were cut and stained with McNeal's tetrachrome for bone volume (BV/TV) and osteoid/osteoblast measures or with TRAP for osteoclast measurements. Mid-sagittal $(8 \mu \mathrm{m})$ sections were also left unstained and used for dynamic histomorphometry measurement of fluorescent calcein; mice received two sequential intraperiotoneal injections 7-14 and 3 days prior to necropsy. To measure differences in femoral bone length between Kal-KO and WT mice, bones were cleaned of tissue and length measured using digital calipers. Measurements were repeated 3 times for the same limb and averaged.

\section{Statistical analysis}

The average and standard error of the mean (SEM) of individual bone parameters were calculated. Statistical significance between Kal-KO mice and control littermate mice was determined with two-way ANOVA using the SYSTAT Software, SPSS Science (Chicago, IL). For all other analyses, statistical significance was determined by standard Student $T$ test. To control for non-parametric data distribution, a log transformation of RANKL serology data was performed prior to statistical analysis by Student $T$-test. A 5\% significance level was used for all tests.

\section{Results}

\section{Kalirin is expressed in osteoclasts and osteoblasts}

We determined whether the Rho-GEF Kalirin was expressed in osteoclasts by Western blotting with an antibody to the spectrin-repeat domain, which detects many of the Kalirin isoforms. Osteoclasts were differentiated with RANKL and M-CSF from the bone marrow of mice. Western blotting revealed several molecular weight Kalirin species in osteoclasts, including several protein species that corresponded in size to the previously reported Kalirin-7, Kalirin-9 and Kalirin-12 isoforms. Similarly, we performed Western blot analysis using osteoblasts derived from the calvaria of neonatal mice, which also revealed multiple isoforms of Kalirin (Fig. 1B). These initial findings prompted us to determine if Kalirin plays a role in bone cell function and in the regulation of skeletal bone mass.

\section{Deletion of the Kalirin gene decreases bone mass and bone remodeling}

To examine the potential role of Kalirin in the regulation of bone mass and bone geometry, we performed micro-CT analysis of Kalirin knockout (Kal-KO) which lack exon 13 of the Kalrn gene [12]. Since exon 13 of the Kalrn gene encodes the spectrin repeat common to all 
major Kalirin isoforms, as well as several N-terminal splice variants, global Kal-KO $\left(\mathrm{Kal}^{-1-}\right)$ mice lack detectable Kalirin-7, Kalirin-9 and Kalirin-12 in all tissues [17,18]. Deletion of Kalirin isoforms in Kal-KO mice, but not littermate wild-type (WT, $\mathrm{Kal}^{+/+}$) controls was confirmed by PCR (Fig. 1C) as previously reported [17,18]. Western blotting also confirmed deletion of Kalirin in osteoclasts and osteoblasts from Kal-KO mice (Fig. $1 \mathrm{C})$.

Micro-CT analysis of femoral trabecular bone from adult 14 week old mice revealed a dramatic $44 \%$ lower trabecular bone volume to total volume (BV/TV) in female Kal-KO mice, compared to WT sex-matched littermates (Fig. 2). In addition, female Kal-KO mice exhibited lower trabecular thickness $(-25 \%)$ and trabecular number $(-35 \%)$, compared to WT mice. We also examined male mice and found reduced BV/TV $(-19 \%)$ and TbN $(-15 \%)$, but not TbTh in male Kal-KO mice, compared to male WT mice. Micro-CT analyses of cortical bone geometry also showed a phenotype consistent with decreased trabecular bone in Kal-KO mice. Specifically, female Kal-KO mice exhibited significantly lower cortical area (-14.4\%) and polar cross-sectional moment of inertia (CSMI) (-28\%), but no change in cortical thickness or perimeter, compared to female WT mice (Fig. 3). Male Kal-KO mice exhibited a decrease in cortical area (-13.3\%), cortical perimeter $(-19 \%)$ and CSMI (-28.5\%), but cortical thickness was similar between male Kal-KO mice and WT littermates. Together, our studies reveal that deletion of Kalirin in mice leads to a decrease in trabecular and cortical bone mass, with female mice exhibiting lower trabecular bone parameters than male Kal-KO mice.

We next investigated the role of Kalirin in bone remodeling. Dynamic histomorphometric analysis of distal femurs from 14 week old Kal-KO and WT littermate mice revealed a $20 \%$ lower mineral surface/bone surface (MS/BS) and bone formation rate (BFR) in female KalKO mice, compared to female WT mice. In contrast, mineral apposition rate (MAR) was unchanged (Fig. 4). Furthermore, we found no difference in BFR, MS/BS or MAR in male Kal-KO mice, compared to male WT mice of the same age.

\section{Kalirin regulates osteoclast and osteoblast number in vivo}

The decreased BV/TV and BFR in female Kal-KO mice suggested a defect in osteoclast and/or osteoblast number/activity in vivo. To gain insight into the role of Kalirin on bone cells in vivo, we performed histological analysis of Kal-KO and WT mice. In agreement with micro-CT analyses, Von Kossa staining revealed decreased mineralized surface in KalKO mice (Fig. 5A). Histological analyses of stained femoral bone sections revealed a $46 \%$ decrease in the number of osteoblasts per bone surface (Ob.N/BS) in female Kal-KO mice, compared to female littermate WT mice (Fig. 5B). Furthermore, we found a dramatic increase in osteoclast surface to bone surface (Oc.S/BS) in female Kal-KO mice compared with WT littermates (Figs. 5C and D). In contrast, Ob.N/BS was increased by $45 \%$ in male Kal-KO mice whereas Oc.S/BS remained unchanged, relative to male WT mice.

\section{Deletion of Kalirin affects longitudinal bone mass}

Given that Kal-KO mice exhibited decreased bone mass at 14 weeks of age, we examined if Kalirin deletion affected bone mass in younger ( 8 week-old) and/or older mice (36 weekold) (Figs. 6A-F). Similar to 14 week-old mice, micro-CT analysis of 8 week-old female Kal-KO mice revealed a significantly lower BV/TV $(-35 \%)$, compared to age-matched WT mice. Tb.Th. $(-13 \%)$ and Tb.N. $(-26 \%)$ were also lower in 8 week-old female Kal-KO mice. We next examined if trabecular bone parameters were reduced in 36 week-old mice. Female 36 week-old Kal-KO mice also exhibited decreased BV/TV (-57\%) compared with age-matched WT mice (Fig. 6D). In addition, Tb.N. was lower (-50\%) in female Kal-KO mice than WT mice, whereas Tb.Th. was unchanged. We also performed micro-CT analysis 
of 8 and 36 week-old male Kal-KO mice. Although we found decreased BV/TV $(-27 \%)$ and Tb.N (-25\%) in 8 week-old male Kal-KO mice, Tb.Th was similar to 8 week-old WT mice (Figs. 6A-C). In contrast, only Tb.N (-23\%) was reduced in 36 week-old male Kal-KO mice compared with age-matched WT mice (Figs. 6D-F).

Trabecular bone architecture deteriorates throughout life and is more pronounced in females than males [19]. Therefore, we calculated the $\%$ decrease in trabecular bone parameters (BV/TV, Tb.Th and Tb.N) for Kal-KO mice at 8, 14 and 36 weeks of age (Figs. 6G-I). Consistent with C57BL/6J mice [19], BV/TV was decreased with increasing age of both WT and Kal-KO mice. In female mice, we found that the \% difference in BV/TV between 36 week-old Kal-KO and WT mice was much greater than the \% difference for 8 and 14 weekold mice (indicated by negative slope) (Fig. 6G). In addition, 36 week-old Kal-KO mice displayed lower Tb.N (\% decrease) than 8 and 14 week-old mice (Fig. 6I). However, no change in \% decrease for $\mathrm{Tb}$.Th. was found in female Kal-KO mice with increasing age (Fig. 6H). In males, BV/TV was slightly higher at 36 week-old mice than 8 week-old mice. We also found a greater decrease in Tb.N. in 36 week-old Kal-KO mice compared to 8 and 14 week-old mice, whereas Tb.Th. was similar in male Kal-KO mice at all ages (Fig. 6H).

To determine if Kalirin potentially regulated bone growth, we examined bone length of KalKO and WT femurs at 8,14 and 36 weeks of age. Although 8 and 38 week-old Kal-KO mice displayed similar femoral length to WT mice, a significant decrease in bone length was observed for 14 week-old mice (Fig. 6J). No change in bone length was found for male mice at any of the ages examined.

\section{Analysis of serum biomarkers in Kal-KO mice}

We collected whole blood from 8, 14 and 36 week old Kal-KO and WT mice and then quantified the level of several biomarkers in serum (Supplementary Fig. 1). However, we found no significant changes in the circulating levels of RANKL or OPG in either female or male Kal-KO mice, regardless of the age of the mice. We also examined sera for collagen Cterminal telopeptides (CTX), a marker of osteoclast bone resorbing activity, and surprisingly found no detectable change in Kal-KO mice for all age groups. Consistent with CTX, we found no change in the level of tartrate-resistant acid phosphatase (TRAP) a marker of osteoclast number in 14 week-old Kal-KO and WT mice. Furthermore, serum levels of osteocalcin and pro-collagen type I N-terminal propeptide (P1NP), markers of osteoblast activity and collagen metabolism, respectively [20] were unchanged in female or male Kal$\mathrm{KO}$ mice at 14 weeks of age. Since bone length was reduced in Kal-KO female mice at 14 weeks of age, we investigated the possible physiological reason for the decreased bone length by measuring serum levels of insulin-like growth factor-1, which is a known regulator of bone growth. Consistent with the bone length data, we found decreased levels of IGF-1 only in 14 week-old female Kal-KO mice (Fig. 6K and Supplementary Fig. 1). No change in IGF-1 was observed in 8 or 36 week old female mice or in male mice at all ages tested. These findings suggest that Kalirin may control bone growth in female mice, via decreasing serum levels of IGF-1.

\section{Deletion of Kalirin affects the differentiation and activity of osteoclasts and osteoblasts}

To investigate the cellular mechanism for the reduced trabecular and cortical bone parameters in Kal-KO mice, we examined if deletion of Kalirin regulated differentiation and function of osteoclasts or osteoblasts in vitro. We first examined osteoclast differentiation in Kal-KO and WT mice. To generate osteoclasts, non-adherent bone-marrow derived hematopoietic cells were differentiated in the presence of M-CSF $(20 \mathrm{ng} / \mathrm{ml})$ and RANKL (40 and $80 \mathrm{ng} / \mathrm{ml}$ ). The number of TRAP-positive multi-nucleated osteoclasts was then quantified. As shown in Fig. 7A, we found a significant increase in the number of 
osteoclasts differentiated from the bone marrow of Kal-KO mice with low and high levels of RANKL (40 and $80 \mathrm{ng} / \mathrm{ml}$ ), suggesting a cell-autonomous effect on the RANKL-mediated differentiation of osteoclasts. We next examined if deletion of Kalirin affected the morphology and/or bone resorbing activity of osteoclasts on dentin in vitro. Osteoclasts from WT or Kal-KO cultures (generated as described above) were replated onto dentin slices for $48 \mathrm{~h}$, and the area resorbed was stained with toluidine blue and quantified. Data was normalized for the number of TRAP-positive cells present on bone chips. Alternatively, osteoclasts were replated onto glass coverslips, labeled for actin and examined microscopically. Interestingly, osteoclasts from Kal-KO mice were significantly more active in bone resorbing activity on dentin than WT osteoclasts (Fig. 7B). However, osteoclast morphology, multi-nucleation and the number of cells exhibiting a peripheral actin-ring appeared similar between Kal-KO and WT osteoclasts (Fig. 7C).

Given that osteoblasts regulate osteoclast differentiation through the RANKL/OPG ratio and the fact that osteoclast number was increased in Kal-KO mice in vivo (Fig. 5B), we examined if osteoblasts from Kal-KO mice supported osteoclast differentiation in vitro (Fig. 7D). For these mix-and-match cultures, equal numbers of non-adherent bone marrowderived cells from WT and Kal-KO mice were co-cultured with equal numbers of osteoblasts from either WT or Kal-KO mice, respectively (Fig. 7D). Interestingly, we observed an increase in the number of osteoclasts generated in co-cultures containing either WT or Kal-KO bone marrow in combination with Kal-KO osteoblasts. In addition, osteoclast numbers were increased when bone marrow from Kal-KO mice was co-cultured with osteoblasts from either WT or Kal-KO mice. Furthermore, osteoclastogenesis was highest when Kal-KO bone marrow was co-cultured with Kal-KO osteoblasts. These findings reveal that deletion of Kalirin in osteoblasts promotes their ability to stimulate osteoclast differentiation.

Finally, we determined whether deletion of Kalirin affected osteoblast differentiation in vitro. Equal numbers of osteoblastic cells from the calvaria of neonatal mice were differentiated in vitro for 7 days in the presence of ascorbic acid and $\beta$-glycerolphosphate.

Alkaline phosphate activity (ALP) was then quantified (Fig. 7E). Alternatively, osteoblastic cells were differentiated for 21 days and mineralization activity was quantified based on the elution of the calcium-binding Alizerin-S red stain (Fig. 7F). In addition, we isolated stromal-derived osteoblasts from WT and Kal-KO mice and differentiated them in osteogenic media for 3-21 days. ALP activity and $\mathrm{Ca}^{2+}$ deposition (mineralizing activity) were then quantitated. These studies revealed a significant increase in the ALP activity of Kal-KO osteoblasts (calvaria and stromal osteoblasts), compared to WT osteoblasts. In contrast, the mineralizing activity of calvarial-derived or stromal-derived osteoblasts from Kal-KO mice was significantly lower than WT mice over 7-21 days. These findings therefore suggest that deletion of Kalirin promotes ALP activity of osteoblasts, but inhibits their ability to mineralize the extracellular matrix.

\section{Regulation of ERK signaling and OPG secretion by Kalirin in osteoblasts}

It is well established that the ratio of RANKL to OPG is a critical determinant of osteoclast differentiation. Therefore, we examined if Kalirin regulated the ratio of secreted RANKL and OPG by osteoblasts. Calvarial OBs from WT and Kal-KO neonatal mice were grown in culture with media containing fetal bovine serum (FBS) and conditioned media was collected after $24 \mathrm{~h}$ and assayed for OPG or RANKL levels by immunoassay (Fig. 8). In some experiments, normal growth media was replaced with media containing $10 \%$ charcoalstripped FBS (CH-FBS), which contains lower levels of hormones and growth factors than normal FBS. As shown in Fig. 8A, Kal-KO osteoblasts cultured in media containing 10\% FBS secreted lower levels of OPG than WT osteoblasts (Fig. 8A). However, there was no 
change in secreted OPG levels when Kal-KO osteoblasts were cultured in media containing CH-FBS. In contrast to OPG, there was no significant difference in secreted RANKL in KalKO and WT osteoblast cultures containing either 10\% FBS or CH-FBS media (Fig. 8B). Nevertheless, as a consequence of decreased OPG secretion, the ratio of RANKL/OPG was increased by $30 \%$ in Kal-KO osteoblasts cultured in the FBS-containing media (Fig. 8C). These findings suggest that unidentified growth factors and/or hormones present in FBS potentially suppress OPG secretion by Kal-KO osteoblasts. We also examined OPG secretion by stromal-derived osteoblasts cultured in media containing 10\% FBS which gave similar results (data not shown), further confirming that Kalirin deletion regulates OPG secretion by osteoblasts.

To begin to understand the role of Kalirin in intracellular signaling leading to changes in OPG secretion, we examined ERK signaling in osteoblasts. Using the same experimental design as above, we asked if ERK (Erk1/2) signaling was altered in calvarial osteoblasts cultured in media containing 10\% FBS or 10\% CH-FBS (Fig. 8D). Compared to WT osteoblasts, phosphorylated ERK was elevated in Kal-KO osteoblasts cultured for $24 \mathrm{~h}$ in media containing FBS but not CH-FBS. We also examined phospho-ERK levels in WT and Kal-KO osteoblasts cultured for up to 6 days in media containing FBS (Fig. 8E).

Interestingly, phospho-ERK expression was increased with increasing time of culture of Kal-KO osteoblasts. We next measured OPG levels in day 2 and day 6 osteoblast cultures (Fig. 8F). Consistent with an increase in ERK signaling, we found decreased secretion of OPG in conditioned media in these cells. To further examine a potential link between ERK signaling and OPG secretion, we examined the effect of the ERK inhibitor U0126 on phospho-ERK protein levels and OPG secretion in Kal-KO and WT osteoblasts. As predicted, U0126 reduced phospho-ERK levels in both WT and Kal-KO osteoblasts (Fig. 8G). U0126 treatment also led to a corresponding increase in OPG secretion by WT and Kal-KO osteoblasts (Fig. 8H), confirming that ERK is a negative regulator of OPG secretion. In addition, inhibition of ERK signaling with U0126 restored OPG secretion by Kal-KO osteoblasts to a level similar to WT osteoblast cultures. We also isolated total protein from long bones of mice and performed Western blotting (Fig. 8I) which also revealed an increase in phosphorylated ERK in Kal-KO long bones. Taken together, these findings demonstrate that deletion of Kalirin promotes ERK signaling in vitro and ex vivo, leading to a decrease in OPG secretion by osteoblasts, and possibly osteocytes, which are the most abundant cell-type in bone.

\section{Discussion}

Bone homeostasis is maintained through the balanced interaction of osteoblasts and osteoclasts, and is affected by multiple paracrine and/or endocrine factors. Abnormalities in the function of the bone cells, or the factors that regulate them, can result in bone loss leading to a variety of skeletal disorders, including osteoporosis. Our study demonstrates for the first time that the Rho-GEF Kalirin is an important regulator of bone remodeling, affecting both trabecular and cortical bone mass. Further, we demonstrate that Kalirin functions in part by regulating the activity of both osteoclasts and osteoblasts.

Micro-CT analyses revealed a low-bone-mass phenotype in Kal-KO mice, with a decrease in trabecular and cortical bone mass observed in both male and female mice. Female Kal-KO mice exhibited a more severe bone loss phenotype than male Kal-KO mice over several ages (Fig. 6), suggesting a sex-specific difference in the magnitude of Kalirin effects. We found that in female Kal-KO mice, bone formation rate and MS/BS were also decreased, but MAR was normal, suggesting that the low-bone-mass phenotype may in part be due to a decrease in osteoblast number, and therefore bone remodeling units in vivo. Indeed, our histomorphometry data confirmed a decrease in Ob.N/BS in 14 week-old female Kal-KO 
mice. Consistent with a decrease in the mineralizing activity of Kal-KO osteoblasts, in vitro studies revealed reduced calcium deposition, as measured by elution of Alizerin-S red staining, in Kal-KO osteoblasts, compared to WT osteoblasts (Figs. 7E-H).

Trabecular architecture increases during bone development but later deteriorates with advancing age. We found that BV/TV and Tb.N were lower in 8 week-old female Kal-KO mice than age-matched WT mice, suggesting that Kalirin may play an early role in bone accretion during growth. Published studies have reported that basal growth hormone $(\mathrm{GH})$ secretion is increased in pituitary cultures from Kal-KO mice [12], suggesting that GH and/ or insulin-like growth factor 1 (IGF-1) may be altered in Kal-KO mice. In support of a potential role for Kalirin in longitudinal bone growth, we found decreased bone length, as well as decreased circulating levels of IGF-1, in 14 week-old Kal-KO female mice, compared to age matched WT mice. However, bone length and IGF-1 levels appeared normal in 8 and 36 week-old Kal-KO mice, as well as in male mice. These findings suggest a temporal and sex-specific effect of Kalirin on bone growth. It is also possible that Kal-KO mice display delayed bone growth leading to a decrease at 14 weeks of age, but that they continue to grow after 14 weeks, resulting in similar bone lengths to WT mice by 36 weeks of age. Our future studies will examine more specifically the role of Kalirin in longitudinal bone growth at different ages and whether Kalirin regulates bone mass in young 1 monthold mice.

Micro-CT analysis revealed a more severe low-bone-mass phenotype in 36 week-old female Kal-KO mice than in younger mice (Fig. 6), suggesting that bone loss was accelerated with age in the absence of Kalirin. In contrast to female mice, the low-bone-mass phenotype of 8 and 14 week male Kal-KO mice did not deteriorate further by 36 weeks of age, but was similar to WT mice. Given that Ob.N/BS was increased by $45 \%$ in 14 week-old Kal-KO male mice (but decreased in female Kal-KO mice), we speculate that increased osteoblast activity in part reversed the bone-loss phenotype of adult male Kal-KO mice. In addition, we cannot yet exclude the possibility that defects in neuromuscular signaling contribute to the low-bone-mass phenotype of male and female Kal-KO mice. Although Kal-KO mice were shown to exhibit some deficits in neuromuscular junction signaling, it is important to note that no sex-specific differences in neuromuscular function were identified [21]. Furthermore, our findings that Kalirin exerts a sex-specific effect on bone mass, BFR and Ob.S/BS, and that Kal-KO osteoblasts exhibit changes in ALP activity, mineralizing activity and OPG secretion all suggest that Kalirin exerts its effect on the skeleton via defects in paracrine and/or endocrine signaling to bone cells. In other studies, it was reported that Kalirin-7, a major Kalirin isoform, was decreased in CA1 pyramidal neurons from ovariectomized rats, but increased by estradiol replacement [22]; an effect mediated by estrogen receptor alpha (ERa) [23]. Similar to the bone phenotype of female Kal-KO mice in our study, it was also reported that female estrogen receptor double KO mice (ERa $\beta \mathrm{KO})$ exhibit dramatically lower trabecular bone volume than male mice, which was associated with a decrease in bone remodeling [24-26]. Taken together, these studies indicate that deletion of Kalirin may potentially affect signaling by the estrogen receptor, or possibly the androgen receptor. Examination of the phenotype of neonatal and young Kal-KO mice, which are currently underway, will likely provide additional insight into the role of Kalirin on bone remodeling, longitudinal bone growth and the role of the sex steroids in male and female Kal-KO mice.

OPG and RANKL are two major cytokines involved in regulating osteoclastogenesis. RANKL acts to promote the differentiation of pre-osteoclasts into mature osteoclasts, while OPG acts as a decoy receptor for RANKL and blocks its ability to promote osteoclast differentiation. Our in vitro studies revealed decreased OPG secretion by Kal-KO osteoblasts, but no change in RANKL. Of interest, dysfunction in OPG secretion was only 
observed when Kal-KO osteoblasts were grown in medium containing 10\% FBS, not in the presence of charcoal-stripped FBS (10\% CH-FBS) which lacks many growth factors, proteins and hormones. Consistent with published reports [27-30], we also found an inverse relationship between ERK activity and OPG levels in Kal-KO osteoblasts. Similar to OPG, we found that the increase in ERK signaling was only apparent in cultures grown in 10\% FBS. These findings suggest the presence of a growth factor, hormone or cytokine in serum that promotes ERK signaling, resulting in reduced OPG secretion by Kal-KO osteoblasts. However, given that phosphorylated ERK was also elevated in Kal-KO long bones in vivo, it is unlikely that changes in ERK signaling contribute significantly to the block in osteoblast differentiation observed in vitro. Rather, these studies suggest that Kalirin regulates ERK signaling pathways which control OPG secretion and the RANKL/OPG ratio in osteoblasts as well as osteocytes, which are the major cell type present in long bone (cortical and trabecular bone) preparations. Since Kalirin activates Rac1, which is important for Wnt/Bcatenin activation [33], it is possible that in the absence of Kalirin, Wnt signaling is suppressed, leading to a down-regulation of OPG secretion. Of further interest, $17 \beta$-estradiol has been shown to dose-dependently increase OPG mRNA and protein levels in human osteoblasts expressing the estrogen receptors [22]. In several cell types, estrogen receptor activation has also been shown to stimulate ERK phosphorylation via the RAS-Raf-MEK pathway [24-26]. Whether Wnt or estrogen receptor signaling is disrupted in Kal-KO osteoblasts, leading to increased OPG levels remain to be determined.

Studies examining the role of Kalirin in osteoclasts revealed cell-autonomous and indirect effects of Kalirin on osteoclast differentiation and bone resorption. Osteoclast numbers in vivo and osteoclast differentiation in vitro were both increased in Kal-KO mice. Although osteoclasts appeared morphologically normal with respect to the formation of the sealing zone/podosome belt, we observed a significant increase in osteoclast bone resorbing activity on dentin in vitro (Fig. 7). Although further studies are needed to determine the mechanism of action of Kalirin in osteoclasts, several studies have demonstrated an important role for Rac1, which is activated by Kalirin, on osteoclast motility and bone resorption [3,4]. In addition, it is possible that deletion of Kalirin potentially activates osteoclast anti-apoptotic pathways, promoting survival signaling. Of interest, decreased osteoclast apoptosis is a hallmark of estrogen-deficiency induced osteoporosis. Despite the increase in osteoclastogenesis and bone resorbing activity in vitro and the increase in OCS/BS in vivo, we did not observe a corresponding increase in serum markers of osteoclast activity (CTX) or osteoclast number (TRAP) in Kal-KO mice. A possible explanation may be that changes in the levels of serum biomarkers may have occurred in younger mice. Alternatively, changes in paracrine signaling via osteoblasts may have led to site-specific skeletal changes in osteoclast numbers as a consequence of reduced OPG secretion (increased RANKL/OPG ratio). Given that osteocytes are a major source of RANKL and OPG [31], it is also possible that Kalirin regulates ERK signaling and RANKL/OPG secretion by osteocytes in vivo.

In summary, our studies demonstrate a novel pleiotropic role for the Rho-GEF Kalirin in the cell-autonomous regulation of osteoclast and osteoblast activities, as well as in osteoblastosteoclast crosstalk via changes in OPG secretion. In addition, our findings indicate that Kalirin may act in part by altering paracrine signaling in vivo, leading to changes in bone remodeling and bone mass. Finally, our studies reveal a sexual dimorphic skeletal phenotype in Kal-KO mice, with more severe bone loss in female mice, suggesting that Kalirin may be important in the regulation of bone mass by steroid hormones. Future studies, including the generation of conditional knockout mice, examining young growing mice and examining sex-hormone signaling will be necessary to better understand the mechanism of action of Kalirin. 


\section{Supplementary Material}

Refer to Web version on PubMed Central for supplementary material.

\section{Acknowledgments}

This work was funded in part by an Indiana University Collaborative Research Grant (IU-CRG) from the Office of the Vice President for Research (AB), a Postdoctoral Research Fellowship Grant and other funds from the Indiana University School of Dentistry (AB). Funding for this work was also provided in part by an NIH grant (\#AR060332) to AB and NIH DK-32948 grant to REM and BAE. We sincerely thank Dr. Sara Windahl, Dr. Jack Martin, Dr. Natalie Sims and Dr. Russell Main for their helpful discussions. We also thank Dr. David Burr for critically evaluating this manuscript. We thank Cameron Carter, Anissa Mahmoudi, Ajaydeep Singh and Neelam Shah for laboratory assistance, and Drew Brown assistance with micro-CT analyses. We also thank George Eckert for statistical support and Julie Daley and Kathy Sterrett for administrative assistance.

\section{References}

1. NOF clinician's guide to prevention and treatment of osteoporosis. National osteoporosis foundation. 2012. www.nof.org

2. Bos JL, Rehmann H, Wittinghofer A. GEFs and GAPs: critical elements in the control of small G proteins. Cell. 2007; 129:865-77.

3. Itokowa T, Zhu ML, Troiano N, Bian J, Kawano T, Insogna K. Osteoclasts lacking Rac2 have defective chemotaxis and resorptive activity. Calcif Tissue Int. 2011; 88:75-86.

4. Bourne, HR. ADP-ribosylating toxins and G proteins. In: Joel, Moss; Martha, Vaughan, editors. Insights into signal transduction. Washington, DC: American Society for Microbiology; 1990.

5. McPherson CE, Eipper BA, Mains RE. Genomic organization and differential expression of Kalirin isoforms. Gene. 2002; 284:41-51.

6. McPherson CE, Eipper BA, Mains RE. Kalirin expression is regulated by multiple promoters. J Mol Neurosci. 2004; 22:51-62.

7. Penzes P, Johnson RC, Kambampati V, Mains RE, Eipper BA. Distinct roles for the two Rho GDP/ GTP exchange factor domains of Kalirin in regulation of neurite growth and neuronal morphology. J Neurosci. 2001; 21:8426-34. [PubMed: 11606631]

8. Ma XM, Huang J, Wang Y, Eipper BA, Mains RE. Kalirin, a multifunctional Rho guanine nucleotide exchange factor, is necessary for maintenance of hippocampal pyramidal neuron dendrites and dendritic spines. J Neurosci. 2003; 23:10593-603.

9. Ferraro F, Ma XM, Sobota JA, Eipper BA, Mains RE. Kalirin/Trio Rho guanine nucleotide exchange factors regulate a novel step in secretory granule maturation. Mol Biol Cell. 2007; 18:4813-25. [PubMed: 17881726]

10. Xie Z, Srivastava DP, Photowala H, Kai L, Cahill ME, Woolfrey KM, et al. Kalirin-7 controls activity-dependent structural and functional plasticity of dendritic spines. Neuron. 2007; 56:64056.

11. Ma XM, Kiraly DD, Gaier ED, Wang Y, Kim EJ, Levine ES, et al. Kalirin-7 is required for synaptic structure and function. J Neurosci. 2008; 28:12368-82.

12. Mandela P, Yankova M, Conti LH, Ma X, Grady J, Eipper BA, et al. Kalrn plays key roles within and outside of the nervous system. BMC Neurosci. 2012; 13:136.

13. Kacena MA, Eleniste PP, Cheng YH, Huang S, Shivanna M, Meijome TE, et al. Mega-karyocytes regulate expression of Pyk2 isoforms and caspase-mediated cleavage of actin in osteoblasts. J Biol Chem. 2012; 287:17257-68.

14. Udagawa N, Takahashi N, Akatsu T, Tanaka H, Sasaki T, Nishihara T, et al. Origin of osteoclasts: mature monocytes and macrophages are capable of differentiating into osteoclasts under a suitable microenvironment prepared by bone marrow-derived stromal cells. Proc Natl Acad Sci U S A. 1990; 87:7260-4. [PubMed: 2169622]

15. Arai F, Miyamoto T, Ohneda O, Inada T, Sudo T, Brasel K, et al. Commitment and differentiation of osteoclast precursor cells by the sequential expression of c-Fms and receptor activator of nuclear factor kappaB (RANK) receptors. J Exp Med. 1999; 190:1741-54. [PubMed: 10601350] 
16. Parfitt AM, Han ZH, Palnitkar S, Rao DS, Shih MS, Nelson D. Effects of ethnicity and age or menopause on osteoblast function, bone mineralization, and osteoid accumulation in iliac bone. $\mathrm{J}$ Bone Miner Res. 1997; 12:1864-73.

17. Mandela P, Ma XM. Kalirin, a key player in synapse formation, is implicated in human diseases. Neural Plast. 2012; 2012:728161. [PubMed: 22548195]

18. Samoszuk M, Leuther M, Hoyle N. Role of serum P1NP measurement for monitoring treatment response in osteoporosis. Biomark Med. 2008; 2:495-508.

19. Glatt V, Canalis E, Stadmeyer L, Bouxsein ML. Age-related changes in trabecular architecture differ in female and male C57BL/6 J mice. J Bone Miner Res. 2007; 22:1197-207. [PubMed: 17488199]

20. Janckila AJ, Neustadt DH, Nakasato YR, Halleen JM, Hentunen T, Yam LT. Serum tartrateresistant acid phosphatase isoforms in rheumatoid arthritis. Clin Chim Acta. 2002; 320:49-58.

21. Mandela P, Yankova M, Conti LH, Ma XM, Grady J, Eipper BA, et al. Kalrn plays key roles within and outside of the nervous system. BMC Neurosci. 2012; 13:136.

22. Onoe Y, Miyaura C, Ohta H, Nozawa S, Suda T. Expression of estrogen receptor beta in rat bone. Endocrinology. 1997; 138:4509-12.

23. Ma XM, Wang Y, Ferraro F, Mains RE, Eipper BA. Kalirin-7 is an essential component of both shaft and spine excitatory synapses in hippocampal interneurons. J Neurosci. 2008; 28:711-24. [PubMed: 18199770]

24. Driggers PH, Segars JH. Estrogen action and cytoplasmic signaling pathways. Part II: the role of growth factors and phosphorylation in estrogen signaling. Trends Endocrinol Metab. 2002; 13:422-7.

25. Segars JH, Driggers PH. Estrogen action and cytoplasmic signaling cascades. Part I: membraneassociated signaling complexes. Trends Endocrinol Metab. 2002; 13:349-54. [PubMed: 12217492]

26. Gilad LA, Bresler T, Gnainsky J, Smirnoff P, Schwartz B. Regulation of vitamin D receptor expression via estrogen-induced activation of the ERK 1/2 signaling pathway in colon and breast cancer cells. J Endocrinol. 2005; 185:577-92.

27. Koons A, Crandall M, An GC, Shapiro MB, Kramer S, West MA. Even ephemeral endotoxin exposure establishes endotoxin tolerance. J Trauma. 2008; 64:938-42.

28. Kusumi A, Kusumi T, Miura J, Tateishi T. Passage-affected competitive regulation of osteoprotegerin synthesis and the receptor activator of nuclear factor-kappaB ligand mRNA expression in normal human osteoblasts stimulated by the application of cyclic tensile strain. $\mathrm{J}$ Bone Miner Metab. 2009; 27:653-62. [PubMed: 19449178]

29. Grundt A, Grafe IA, Liegibel U, Sommer U, Nawroth P, Kasperk C. Direct effects of osteoprotegerin on human bone cell metabolism. Biochem Biophys Res Commun. 2009; 389:5505.

30. Tsubaki M, Satou T, Itoh T, Imano M, Yanae M, Kato C, et al. Bisphosphonate- and statin-induced enhancement of OPG expression and inhibition of CD9, M-CSF, and RANKL expressions via inhibition of the Ras/MEK/ERK pathway and activation of p38MAPK in mouse bone marrow stromal cell line ST2. Mol Cell Endocrinol. 2012; 361:219-31.

31. Moriishi T, Fukuyama R, Ito M, Miyazaki T, Maeno T, Kawai Y, et al. Osteocyte network; a negative regulatory system for bone mass augmented by the induction of Rankl in osteoblasts and Sost in osteocytes at unloading. PLoS One. 2012; 7:e40143.

32. Sims NA, Dupont S, Krust A, Clement-Lacroix P, Minet D, Resche-Rigon M, et al. Deletion of estrogen receptors reveals a regulatory role for estrogen receptors-beta in bone remodeling in females but not in males. Bone. 2002; 30:18-25.

33. Wu X, Tu X, Joeng KS, Hilton MJ, Williams DA, Long F. Rac1 activation controls nuclear localization of beta-catenin during canonical Wnt signaling. Cell. 2008; 133:340-53. 
A

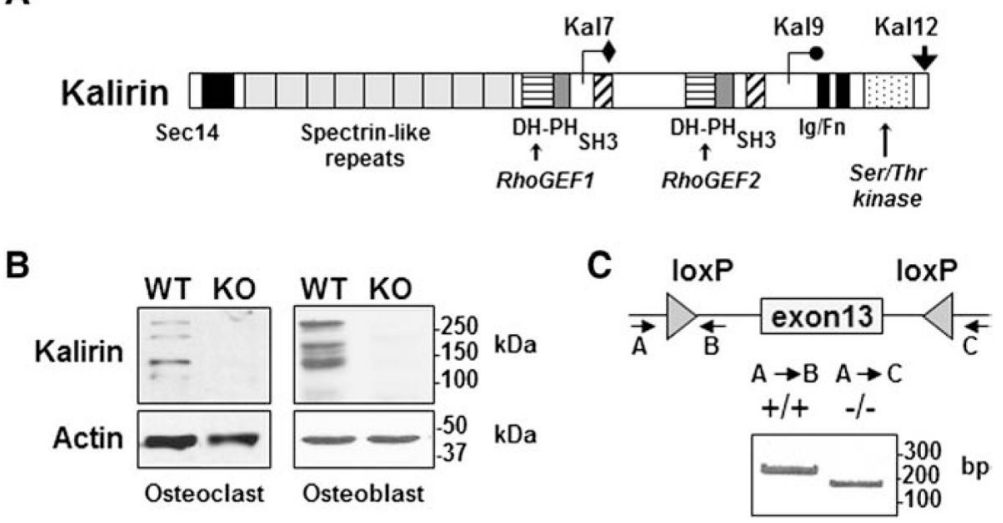

Fig. 1.

Expression of Kalirin in osteoclasts and osteoblasts. (A) Schematic representation of the three major Kalirin isoforms formed by alternative mRNA splicing. All full-length isoforms contain a Sec14 lipid binding domain, nine spectrin repeats and a Rho-GEF (PH-DH) domain. Kal9 also contains an SH3 domain and the second GEF domain. Kal12 contains two SH3 domains and a second GEF domain as well as an Ig/FnIII domain and Ser/Thr kinase domain. (B) Osteoclasts were differentiated from non-adherent bone marrow cells in the presence of MCSF and RANKL, while osteoblasts were obtained from cultures of neonatal calvaria preparations. Kalirin isoforms were identified by Western blotting using an antibody that detects the spectrin domain which is common to each isoform [32]. Protein lysates were prepared from WT mice and from mice lacking Kalirin (Kal-KO). Actin was used as a protein loading control. Molecular mass markers are indicated. (C) Global Kal-KO mice were generated by crossing Kalrn exon 13 floxed mice with Cre-Hprt mice to excise Exon 13 of the Kalirin gene [31]. PCR analysis of rat tail DNA was used to confirm deletion of Kalirin mRNA in mice. The migration of PCR products is indicated by the DNA ladder (base pairs; bp). Western blotting (B) also confirmed the absence of Kalirin isoforms in Kal$\mathrm{KO}$ osteoclasts and osteoblasts. 


\section{A}
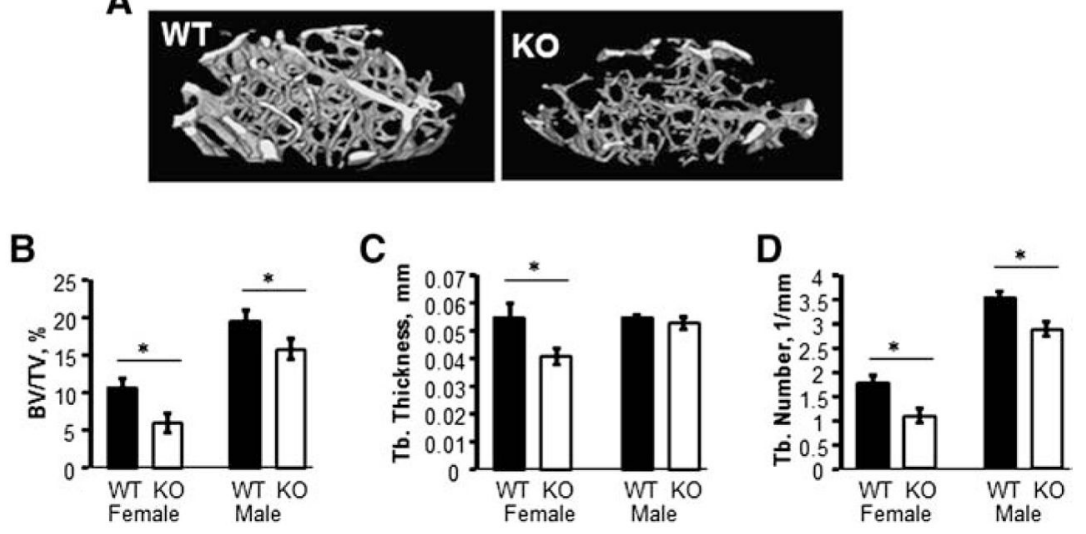

Fig. 2.

Trabecular bone mass is dramatically decreased in Kal-KO mice. (A) Three-dimensional reconstruction of micro-CT images of distal femurs of 14 week old female mice. (B) Trabecular bone parameters of 14 week female (WT and Kal-KO) mice and male (WT and Kal-KO) mice ( $n=6,7,11$ and 9 mice, respectively). Bone volume/total volume (BV/TV), thickness $(\mathrm{TbTh})$ and trabecular number $(\mathrm{TbN})$ were determined. Results shown are mean \pm SEM relative to sex-matched controls. Statistically significance $(p<0.05)$ compared to sexmatched WT mice is indicated by an asterisk. 
A

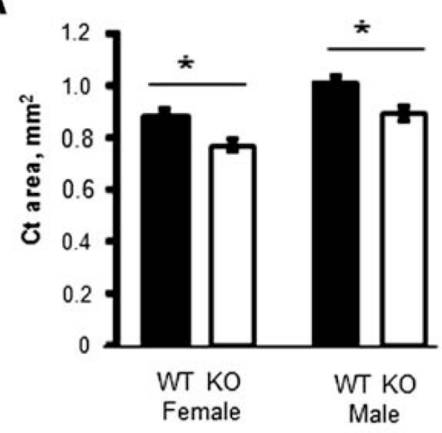

C

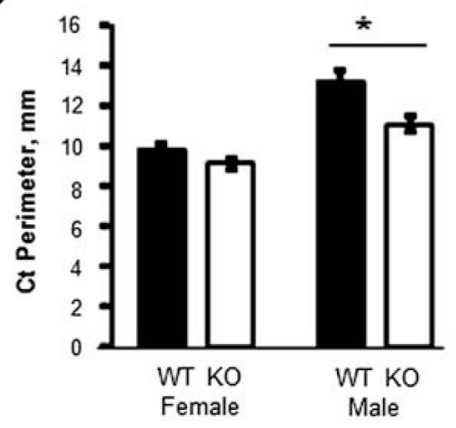

B

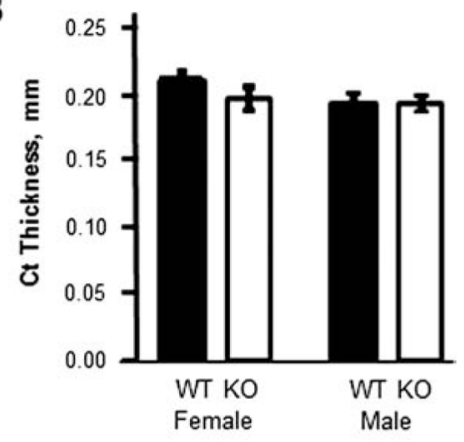

D

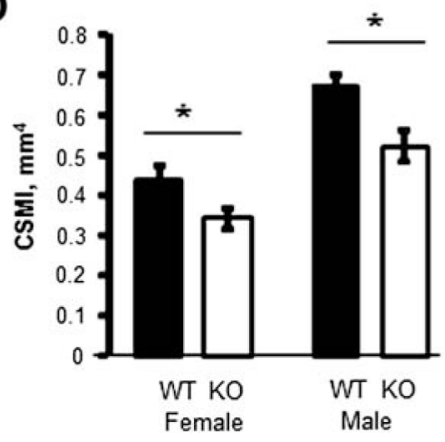

Fig. 3.

Micro-CT analysis of cortical bone geometry. The following cortical bone parameters were determined by micro-CT using 14 week-old mice WT and Kal-KO mice; cortical area (A), thickness (B), perimeter (C) and cross-sectional moment of inertia (CSMI) (D). Graphs are mean \pm SEM of results from female mice $(\mathrm{n}=6 \mathrm{WT}, \mathrm{n}=7 \mathrm{KO})$ and male mice $(\mathrm{n}=11 \mathrm{WT}$, $\mathrm{n}=9 \mathrm{KO})$. Asterisks indicate $\mathrm{p}<0.05$. 
A

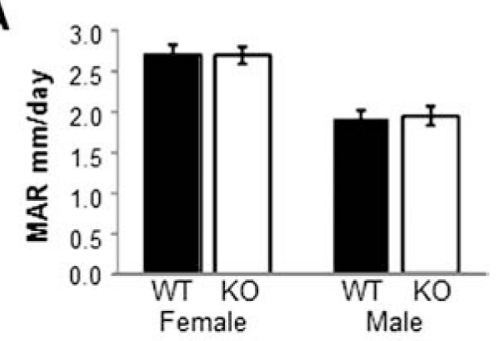

B
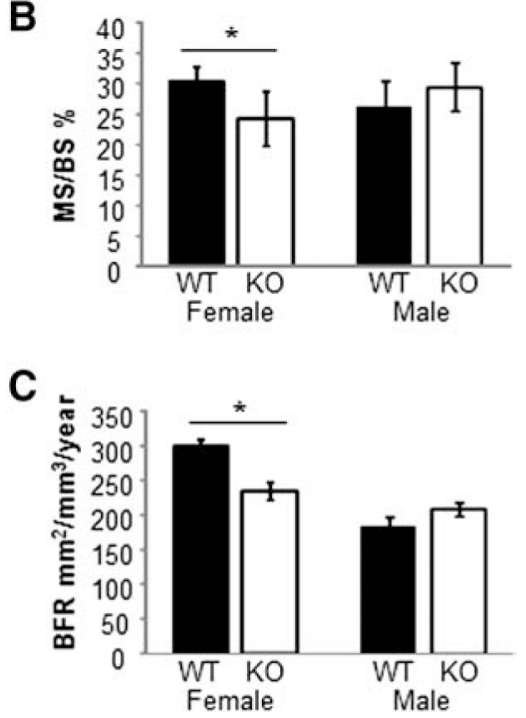

Fig. 4.

Dynamic bone parameters. (A-C) Calcein-labeled femurs from WT and Kal-KO mice were used to calculate the mineral apposition rate (MAR), mineralizing surface per bone surface (MS/BS) and the bone formation rate (BFR). Graphs shown are mean \pm SEM as follows for female mice $(\mathrm{n}=10 \mathrm{WT}, \mathrm{n}=7 \mathrm{KO})$ and male mice $(\mathrm{n}=12 \mathrm{WT}, \mathrm{n}=13 \mathrm{KO})$. 
A

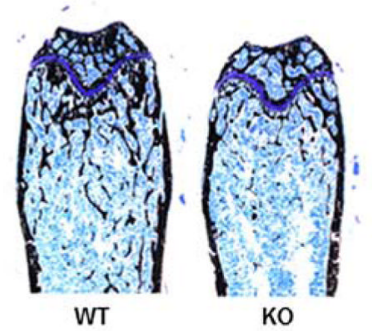

C

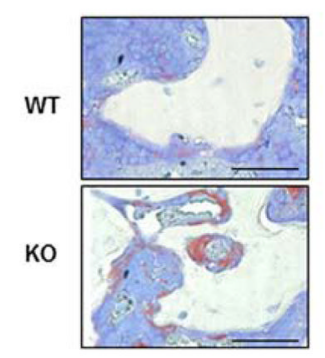

B

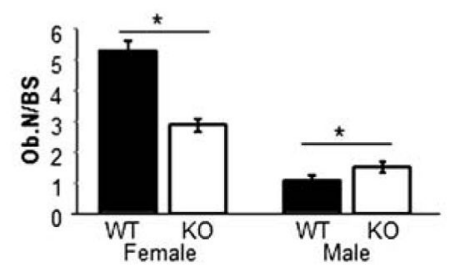

D

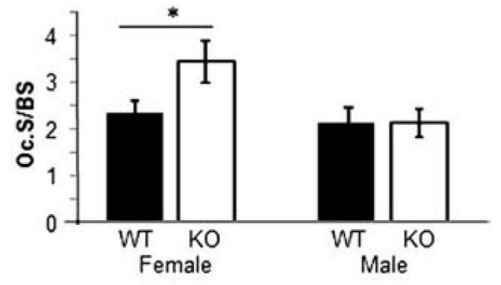

Fig. 5.

Histological analysis of femoral bone. (A) Representative montaged images of histological sections taken from the distal femur of WT and Kal-KO mice labeled with von Kossa and McNeal tetrachrome stains. 10× magnification. (B) The number of osteoblasts associated with trabecular surfaces was quantified and normalized to bone surface (Ob.N/BS). Results represent 3 serial sections per bone (females, $n=3$; males, $n=4-5$ mice per genotype). (C) Tissue sections from WT and Kal-KO femurs were stained for TRAP and counter stained with toluidine blue. TRAP-positive osteoclasts appear purple in color. Scale bar is $50 \mu \mathrm{m}$. (D) The area of TRAP-positive osteoclasts per bone surface (Oc.S/BS) was quantified on 3 serial sections per bone, which revealed an increase in Oc.S/BS in female Kal-KO mice ( $\mathrm{n}=$ 6 mice per genotype), but not in male Kal-KO mice ( $n=8$ mice per genotype), when compared to sex-matched WT mice. 


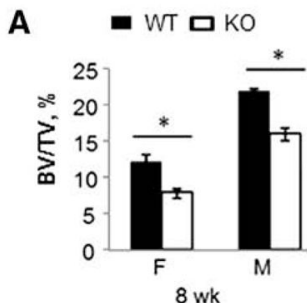

B

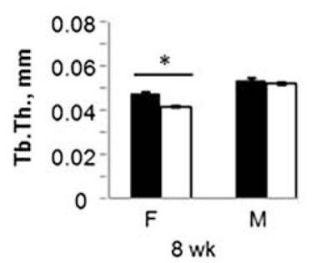

C

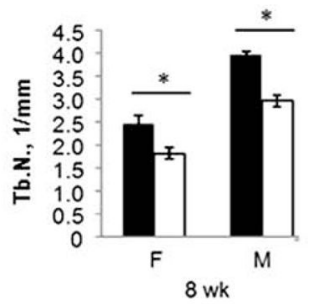

$\mathbf{J}$
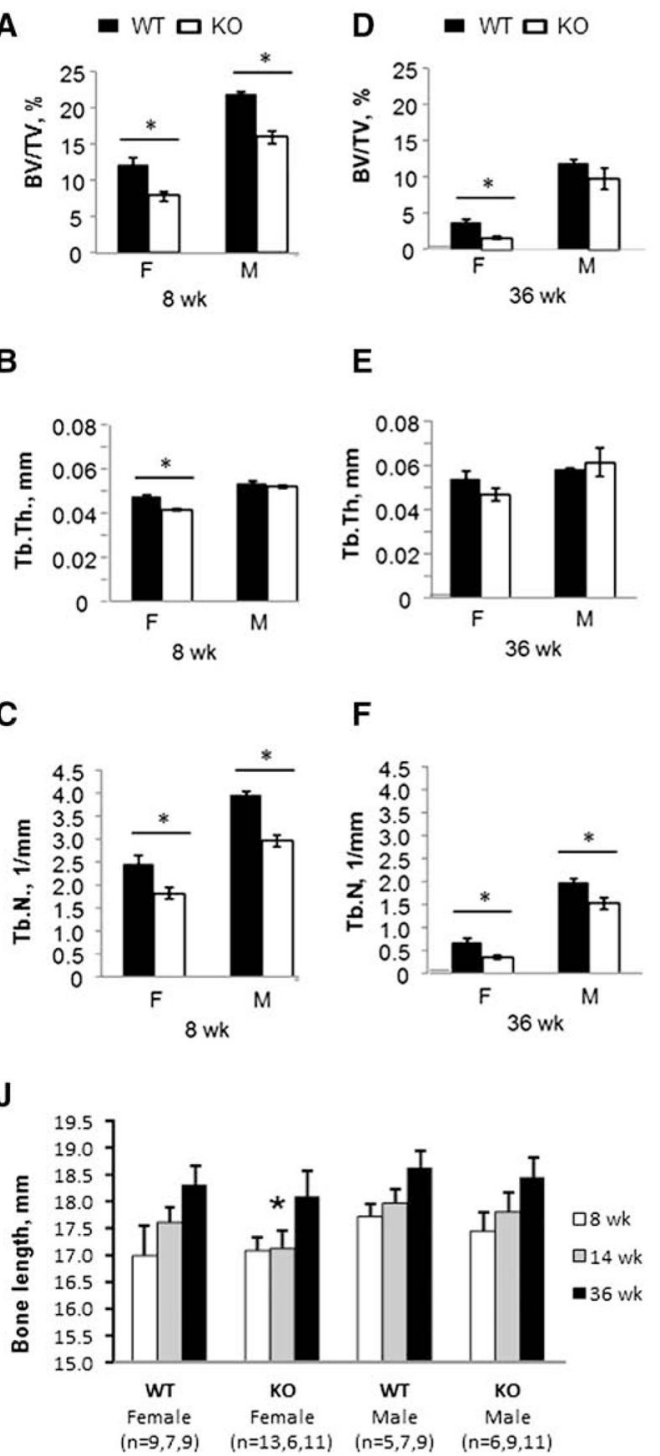

E

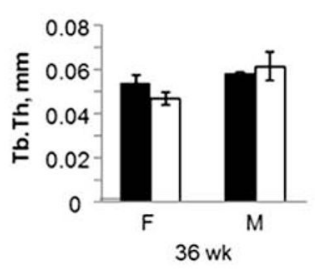

$\mathbf{F}$

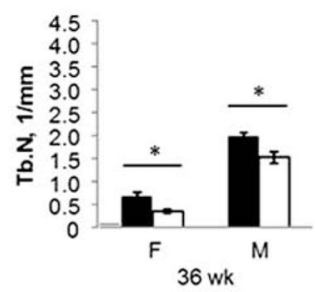

K
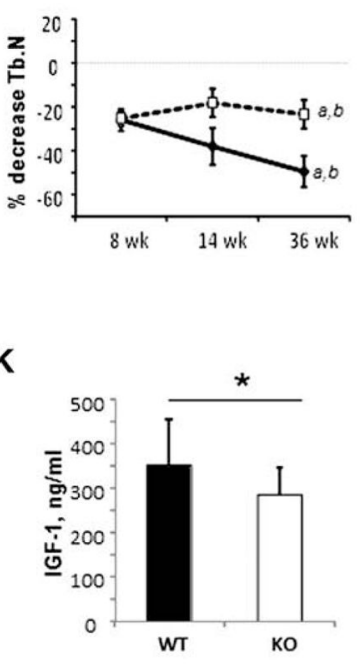

Fig. 6.

Longitudinal effects of Kalirin deletion on bone mass. (A-C) Trabecular bone analysis of 8 week-old female Kal-KO and WT mice $(\mathrm{n}=5,5)$ and male Kal-KO and WT mice $(\mathrm{n}=3,11)$. BV/TV, Tb.Th and Tb.N were determined by micro-CT. Statistically significance $(\mathrm{p}<0.05)$ compared to sex-matched WT mice is indicated by an asterisk. (D-F) Trabecular bone analysis of 36 week-old female Kal-KO and WT mice $(\mathrm{n}=7,8)$ and male Kal-KO and WT mice $(n=8,6)$. The asterisk indicates statistically significance $(\mathrm{p}<0.05)$ compared to sexmatched WT mice. (G-I) The difference in BV/TV between age- and sex-matched Kal-KO and WT mice was determined and expressed as a percentage relative to WT mice. The numbers of 8 and 36 week-old mice was the same as above. For 14 week-old mice we used female WT and Kal-KO mice $(n=7,6)$ and male WT and Kal-KO mice $(n=6,5)$. Statistical significance was determined using 2-way Anova and " $a$ " indicates $\mathrm{p}<0.05$ for the comparison 36 versus 8 week-old mice, " $b$ " indicates $\mathrm{p}<0.05$ for 36 versus 14 week-old mice. (J). Digital calipers were used to measure the length of femoral bones from WT and Kal-KO mice (mm). (K). Serum levels of IGF-1 in 14 week-old female WT $(\mathrm{n}=13)$ and 
Kal-KO $(\mathrm{n}=21)$ mice (see Supplementary figure for additional data). All results shown are mean \pm SEM. 
A

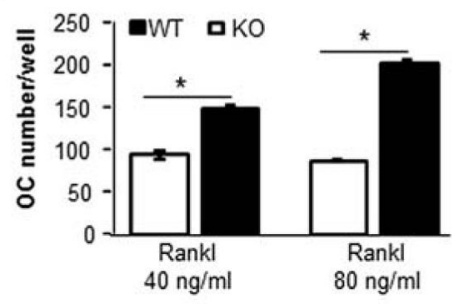

C

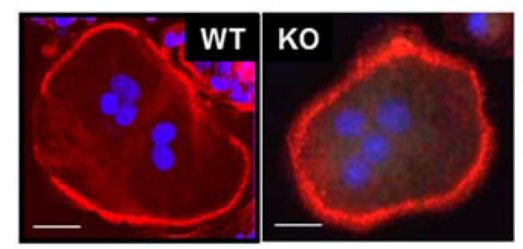

B

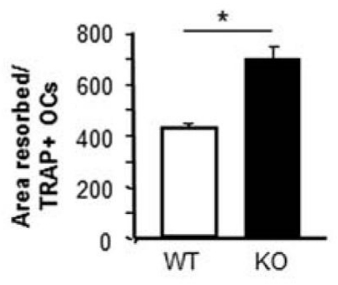

D

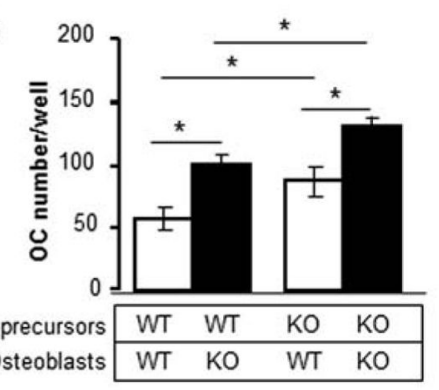

E

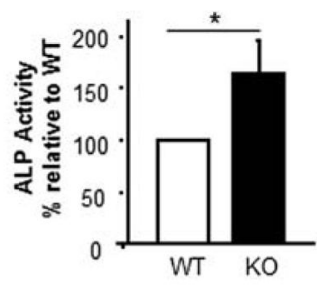

G

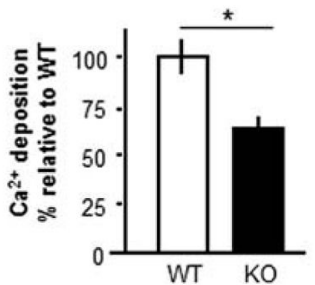

$\mathbf{F}$

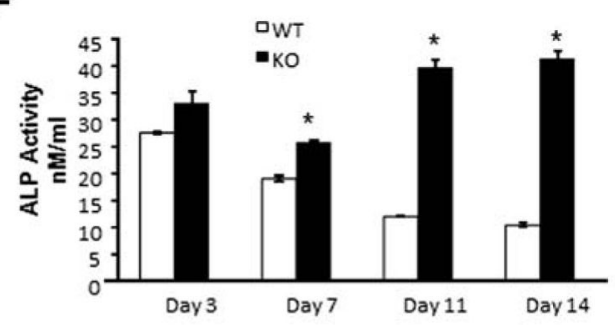

H

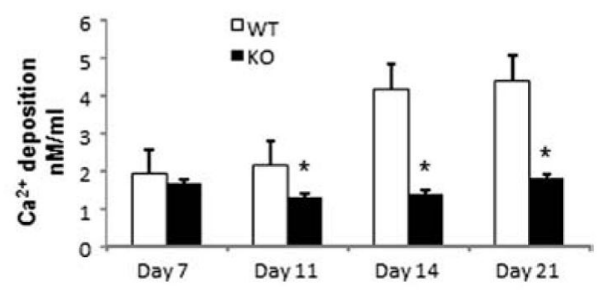

Fig. 7.

Loss of Kalirin affects osteoclast and osteoblast activity. (A) Osteoclasts from Kal-KO and WT mice were differentiated with M-CSF (20 ng/ml) and RANKL (40 or $80 \mathrm{ng} / \mathrm{ml}$ ) for 7 days, and TRAP-positive multinucleated osteoclasts were enumerated. (B) Osteoclasts were differentiated with RANKL and M-CSF and when mature (containing 3 or more nuclei) were replated onto dentin slices for $48 \mathrm{~h}$. The area resorbed was stained with toluidine blue and measured using Bioquant software. The total area resorbed per dentin slice ( $n=4$ /group) was normalized for the number of TRAP-positive osteoclasts. $p<0.05$. (C) Mature osteoclasts were plated onto glass coverslips and stained with rhodamine phalloidin to detect actin. Nuclei were stained with DAPI. Scale bar is $10 \mu \mathrm{m}$. All experiments were reproduced 3-5 times with similar results and representative results are shown. (D) Equal numbers of calvarial osteoblasts $(\mathrm{OB})$ were co-cultured with non-adherent bone marrow cells (BM) derived from either WT or Kal-KO mice as indicated. Cultures were differentiated with $\mathrm{VitD}_{3}$ and $\mathrm{PGE}_{2}$ for 6 days and the number of TRAP-positive osteoclasts was then enumerated. (E) Equal numbers of calvaria-derived osteoblastic cells from Kal-KO or WT neonates were differentiated in osteogenic media for 7 or 14 days. After 7 days, cultures 
were harvested and assayed for ALP activity. Results represent the average \pm SEM for 10 independent experiments, expressed as a percentage relative to WT osteoblasts. (F) Stromal derived osteoblasts were differentiated in osteogenic media for 3-14 days and assayed for ALP (nmol/ml). Results show that loss of Kalirin increases ALP activity over time. (G) Fourteen day calvarial osteoblast cultures were examined for mineralizing activity using the Alizerin-S red elution assay. Results are the average \pm SEM for four independent experiments. Results are expressed as a percentage relative to WT controls. (H) Stromal osteoblasts were differentiated for 7-21 days and assayed for mineralizing activity (Ca2+; $\mathrm{nmol} / \mathrm{ml}$ ). Samples were prepared in triplicate and averaged. Results reveal that loss of Kalirin inhibits the mineralizing activity of osteoblasts. Asterisks indicate $\mathrm{p}<0.05$ between WT and Kal-KO cells at the same day of differentiation. (For interpretation of the references to color in this figure legend, the reader is referred to the web version of this article.) 
A

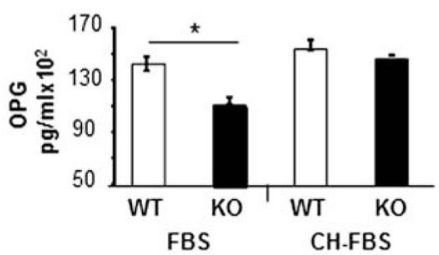

C

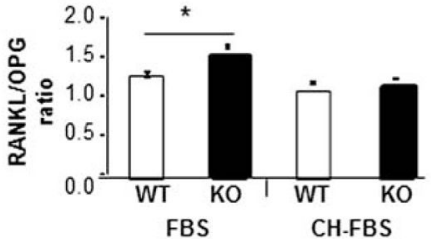

E

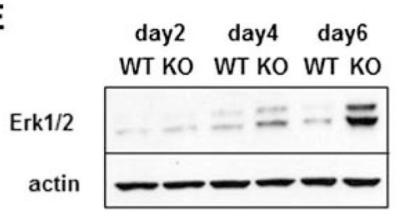

B

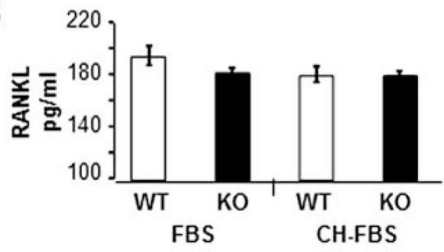

D

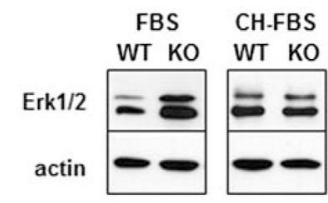

$\mathbf{F}$

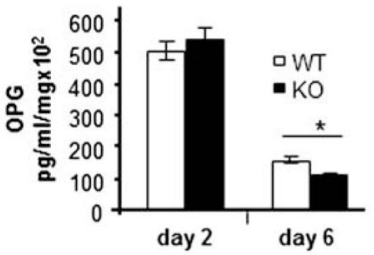

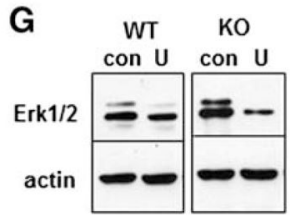

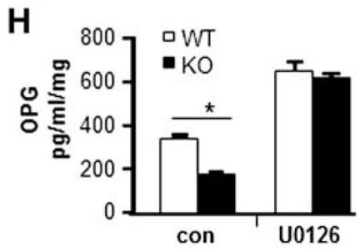

I

actin

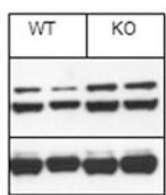

Fig. 8.

Loss of Kalirin affects ERK signaling and OPG secretion. (A-C) Osteoblasts were cultured for 1 week in growth media and then replaced with media containing either 10\% FBS or $10 \% \mathrm{CH}-\mathrm{FBS}$ for $24 \mathrm{~h}$. Conditioned media was collected and assayed for OPG or RANKL, and the ratio of RANKL to OPG was determined as indicated. (D) Cells grown in media containing 10\% FBS or $10 \%$ CH-FBS were lysed and subject to SDS-PAGE and Western blotting with an antibody to phosphorylated ERK1/2. The doublet reflects the p42/p44 isoforms of ERK. Actin was used as a loading control. (E) Osteoblasts from WT and Kal$\mathrm{KO}$ mice were cultured in growth media for 2, 4 or 6 days, followed by Western blot analysis with an antibody to phosphorylated ERK1/2. (F) Media were changed daily and conditioned media collected at day 2 and day 6 were assayed using the OPG immunoassay. (G) Day 6 osteoblast cultures were treated with vehicle or the ERK inhibitor U0126 $(10 \mu \mathrm{M})$ for $24 \mathrm{~h}$, followed by Western blotting for phosphorylated ERK1/2 or actin. $(\mathrm{H})$ Media were collected after $24 \mathrm{~h}$ treatment of osteoblasts with U0126 and assayed for OPG levels. Results were normalized for total protein. Three wells per condition were used and OPG or RANKL was assayed in duplicate. (I) Protein lysates were prepared from long bones after removal of bone marrow (two separate age-matched WT and Kal-KO mice were used). Protein lysates were subject to SDS-PAGE and blotted for phosphorylated Erk1/2. Actin was used as the loading control. All ELISA assay experiments were reproduced at least twice and Western blot experiments were repeated 2-6 times and representative results are shown. 